\title{
Dynamic process of phagocytosis and forms of macrophage cell death induced by ingestion of apoptotic neutrophils
}

\author{
WANG Jiong, HUANG WeiLin, WANG Cheng \& LIU RongYu* \\ Department of Pulmonology, Anhui Geriatrics Institute, the First Affiliated Hospital of Anhui Medical University, Hefei 230022, China
}

Received June 10, 2013; accepted March 4, 2014; published online August 22, 2014

\begin{abstract}
Clearance of apoptotic neutrophils by macrophages is important for both the successful resolution of acute inflammation and homeostasis. However, the dynamic process of phagocytosis of apoptotic neutrophils by macrophages and the fate of macrophages after the ingestion of apoptotic neutrophils has not been well documented. In the present study, we staged the recognition and tethering, internalization, digestion and exocytosis steps of phagocytosis of apoptotic neutrophils. Furthermore, we found that after the ingestion of apoptotic cells, a subset of macrophages underwent cell death by autophagy, apoptosis or oncosis as revealed by transmission electron microscopy and confocal microscopy combined with specific dyes. The percentage of autophagic, apoptotic and oncotic macrophages were $8.00 \% \pm 2.00 \%, 12.33 \% \pm 2.08 \%$, and $3.66 \% \pm 1.50 \%$, respectively. These results indicated that after ingestion of apoptotic neutrophils, a subset of macrophages undergoes autophagy and apoptosis. We propose that autophagy of macrophages after the ingestion of apoptotic cells may be a new mechanism present in the resolution of inflammation.
\end{abstract}

macrophage, neutrophil, apoptosis, phagocytosis, cell death

Citation: Wang J, Huang WL, Wang C, Liu RY. Dynamic process of phagocytosis and forms of macrophage cell death induced by ingestion of apoptotic neutrophils. Sci China Life Sci, 2014, 57: 1018-1023, doi: 10.1007/s11427-014-4726-y

Macrophages and neutrophils are major effector cells in inflammatory diseases. Neutrophils effectively bind and kill microorganisms that might cause damage to tissues, after which they undergo a vigilantly controlled program of cell death known as apoptosis [1-3]. Apoptotic neutrophils are dangerous to their neighboring cells because they may undergo secondary necrosis and release their toxic contents unless they are cleared by professional or non-professional phagocytes. Efficient removal of these intact apoptotic neutrophils by macrophages is believed to be critical for the successful resolution of acute inflammation and the maintenance of tissue homeostasis [4-7]. Previous studies have focused on the recognition and uptake of apoptotic neutrophils by macrophages [6], but the dynamic process of phagocytosis and the fate of macrophages after the ingestion

*Corresponding author (email: rongyuliu@gmail.com) of apoptotic neutrophils have not been well documented.

The murine macrophage-like RAW264.7 cell line has been used to establish phagocytosis assays of apoptotic Jurkat (human T-cell lymphoma) cells and the mouse CTLL-2 cell line [8,9]. To gain a better understanding of the interaction between macrophages and apoptotic neutrophils, and the fate of macrophages after neutrophil ingestion, we analyzed phagocytosis of human apoptotic neutrophils by RAW264.7 cells in real time and focused on the subsequent changes in macrophages after their ingestion of apoptotic neutrophils in vitro.

\section{Materials and methods}

\subsection{Neutrophil isolation and apoptosis induction}

Ten milliliter of heparinized venous blood was obtained 
from healthy volunteers after informed written consent signed by each volunteer and approved by the medical ethic committee of the First Affiliated Hospital of Anhui Medical University. Neutrophils were isolated under sterile conditions using the Percoll discontinuous density gradient centrifugation as previously described, with some modification [10-12]. Briefly, $10 \mathrm{~mL}$ heparinized venous blood was incubated for $5 \mathrm{~min}$ in $50 \mathrm{~mL}$ red blood cell lysis buffer (170 mmol L $\mathrm{NH}_{4} \mathrm{Cl}, 10 \mathrm{mmol} \mathrm{L} \mathrm{KHCO}_{3}, 0.1 \mathrm{mmol} \mathrm{L}^{-1}$ EDTA, $\mathrm{pH}$ 7.3) to remove red blood cells. The remaining cells were layered on the top of a Percoll discontinuous density gradient and centrifuged $(1000 \times g, 30 \mathrm{~min}$, room temperature) without braking. The isolated neutrophils were washed three times with PBS and cultured at a density of $1 \times 10^{6}$ cells $\mathrm{mL}^{-1}$ in Dulbecco's modified Eagle's medium (DMEM; Gibco, Life Technologies, Carlsbad, CA, USA) supplemented with $10 \%$ fetal calf serum (FCS), $2 \mathrm{mmol} \mathrm{L}^{-1}$ glutamine, $100 \mathrm{IU} \mathrm{mL} \mathrm{mL}^{-1}$ penicillin and $100 \mathrm{mg} \mathrm{mL}^{-1}$ streptomycin. Viability and purity of neutrophils isolated using this method were $97 \%$ and $95 \%$, respectively. Spontaneous apoptosis was achieved by culturing the neutrophils for $20 \mathrm{~h}$ at $37^{\circ} \mathrm{C}$ in a humidified incubator with $5 \% \mathrm{CO}_{2}[13,14]$. At this time, the level of apoptosis as assessed by an Annexin V-FITC apoptosis detection kit (BD Biosciences, San Jose, CA, USA) and flow cytometry was $25 \%-60 \%$, whereas necrosis was less than $3 \%$.

\subsection{Live cell imaging of Raw264.7 cells engulfing apoptotic neutrophils and phagocytosis-induced cell death in macrophages}

$2 \times 10^{4}$ Raw264.7 cells were grown overnight at $37^{\circ} \mathrm{C}$ with $5 \% \mathrm{CO}_{2}$ in media consisting of DMEM and $10 \%$ FCS in a $35 \mathrm{~mm}^{2}$ dish. The dish was then placed in a chamber on the stage of an Olympus IX-70 inverted microscope equipped with a CCD camera controlled by SPOT RT software (Diagnostic Instruments, Inc., Sterling Heights, MI, USA). Live cell imaging of phagocytosis was performed as previously described $[13,15,16]$. Approximately $2 \times 10^{3}$ apoptotic neutrophils were added to the dish and allowed to interact with the macrophages. For time-lapse sequences, images were obtained automatically every $30 \mathrm{~s}$ for a period of 5-12 h. The series of images were compiled into digital movies by MetaMorph $^{\mathrm{TM}}$ 7.0 Image Processing software (Universal Imaging Corporation, Downingtown, PA, USA).

\subsection{Transmission electron microscopy}

Macrophages $\left(2 \times 10^{5}\right.$ cells $)$ were transferred to a $35 \mathrm{~mm}$ dish in $1 \mathrm{~mL}$ DMEM with $10 \%$ FCS and allowed to grow overnight before co-incubation with apoptotic neutrophils $\left(4 \times 10^{5}\right.$ cells $)$ at $37^{\circ} \mathrm{C}$ for $60 \mathrm{~min}$. Then the non-ingested neutrophils were removed by vigorous washing. After that RAW264.7 cells were fixed in $2.5 \%$ glutaraldehyde, scraped and pelleted by centrifugation. Ultrathin sections were cut using an ultramicrotome (LKB-4, Stockholm, Sweden). Sections stained with uranyl acetate and lead citrate were observed under a transmission electron microscope (JEM-1230, JEOL Ltd., Tokyo, Japan).

\subsection{Confocal microscopy}

After interacting with cultured neutrophils labeled with 7-AAD for $60 \mathrm{~min}$, macrophages were stained with 0.05 mmol L ${ }^{-1} \mathrm{MDC}$ and $100 \mu \mathrm{g} \mathrm{mL}^{-1} \mathrm{AO}, 100 \mu \mathrm{g} \mathrm{mL}^{-1} \mathrm{AO}$, or $100 \mu \mathrm{g} \mathrm{mL}^{-1} \mathrm{AO}$ and $100 \mu \mathrm{g} \mathrm{mL}^{-1} \mathrm{~EB}$ respectively for $15 \mathrm{~min}[17,18]$. Then the cells were washed three times with PBS and scanned using a Zeiss LSM510 confocal microscope within $1 \mathrm{~h}$. Monodansylcadaverine (MDC; Sigma-Aldrich, St Louis, MO, USA) is a specific dye for the autophagosome, acridine orange (AO; Sigma-Aldrich) is a cell-permeable dye that intercalates into DNA and results in a green color while ethidium bromide (EB; Sigma-Aldrich) enters cells with disrupted membrane integrity and intercalates into RNA and double-stranded DNA to appear orange. Thus, differential uptake and binding of these dyes allow us to identify macrophages that had phagocytosed apoptotic neutrophils and then underwent autophagy, apoptosis or oncosis. In each experiment, at least 100 macrophages that had engulfed 7-AAD labeled apoptotic neutrophils were counted, and the proportion of macrophages with positive granules stained with MDC, AO and EB was expressed as a percentage. Macrophages without interaction with apoptotic neutrophils and stained with AO/MDC, AO and AO/EB were used as corresponding controls.

\subsection{Statistics}

Data are expressed as the mean \pm standard error (SE) and were analyzed for significant differences by the Independent-Samples $T$ Test and One-Way ANOVA with SPSS 10.0 (IBM, Armonk, NY, USA). Differences were considered statistically significant if $P<0.05$.

\section{Results}

\subsection{Live cell imaging of the phagocytosis of apoptotic neutrophils by macrophages and phagocytosis-induced cell death in macrophages}

In time-lapse sequences, we found that most neutrophils appeared to interact actively with the ramified macrophages. The macrophages could recognize and tether the apoptotic neutrophils, followed by engulfment. Analysis of 30 macrophages in 20 independent experiments showed that most macrophages engulfed intact apoptotic neutrophils within a few minutes to $1 \mathrm{~h}$. As shown in Figure 1, we recorded the steps of phagocytosis and the macrophage cell death after ingestion of apoptotic neutrophils. The different stages of 

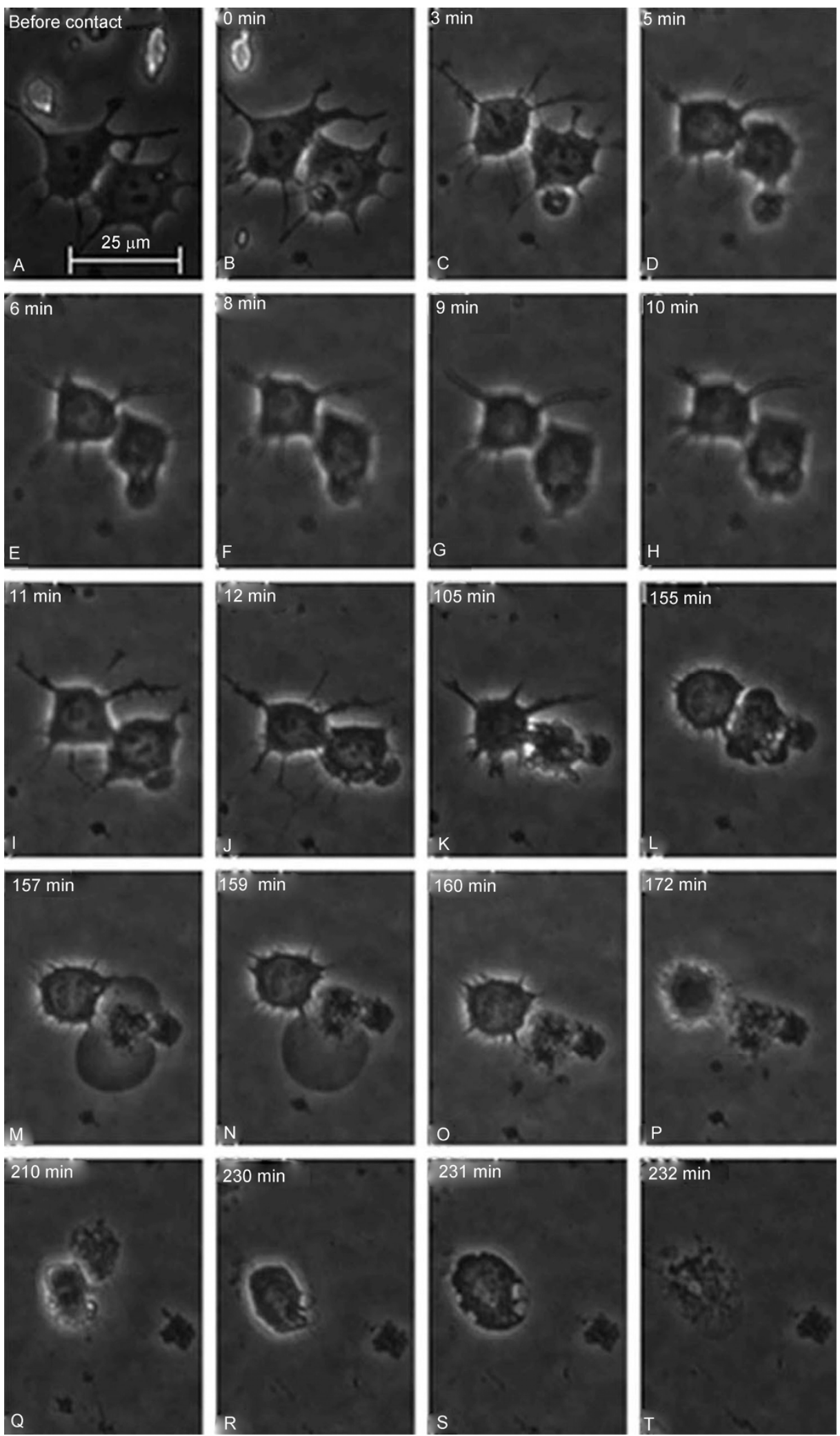

Figure 1 Time-lapse sequence of phagocytosis and macrophage cell death after the ingestion of apoptotic neutrophils. A, Macrophages in culture were highly ramified or branched. B and C, Recognition and tethering of the apoptotic neutrophil: the macrophage interacted with and finally tethered the apoptotic neutrophil. D-F, Internalization of the neutrophil: the macrophage actively protruded pseudopodia to prey the neutrophil and then the two cells membranes fused. G and H, Digestion of the neutrophil. I and J, Exocytosis of the residual neutrophil body. K, Shrinkage of the macrophage after ingestion of the apoptotic neutrophil. L and M, Membrane blebbing of the macrophage. N and O, Membrane rupture of the macrophage. P and Q, Shrinkage of the neighboring macrophage. R-T, Membrane blebbing and rupture of the neighboring macrophage. 
phagocytosis were readily distinguishable. Stage I, recognition and tethering: the apoptotic neutrophil was recognized and finally tethered to the macrophage. This stage lasted about 5 min (Figure $1 \mathrm{~A}-\mathrm{C}$ ). Stage II, internalization: the macrophage initially protruded pseudopodia to capture the neutrophil and the two cells' membranes fused. This lasted about 3 min (Figure 1D-F). Stage III, digestion: the engulfed neutrophil was processed within the macrophage. This stage continued for about $2 \mathrm{~min}$ (Figure $1 \mathrm{G}$ and $\mathrm{H}$ ). Stage IV, exocytosis: the neutrophil became smaller and was excreted out of the macrophage. This stage lasted about 2 min (Figure 1I and J). During the process of phagocytosis, the macrophage was highly active and underwent dramatic structural changes from a ramified cell to a round one.

As time lapsed ( $1 \mathrm{~h}$ later), the macrophage that had ingested the apoptotic neutrophil underwent a characteristic sequence of morphological changes, beginning with cell shrinkage, followed by membrane blebbing, and ultimately concluding in cell membrane rupture (Figure $1 \mathrm{~K}-\mathrm{O}$ ). Subsequently, the neighboring macrophage also underwent cell shrinkage, the membrane blebbing and rupture, just as the preceding one had (Figure 1P-T).

\subsection{Form of macrophage cell death observed by transmission electron microscopy}

To discern the form of macrophage cell death after ingestion of apoptotic neutrophils, we performed transmission electron microscopy. Cells characterized by depletion of organelles with intact non-pyknotic nuclei and autophagosomes were identified as autophagic. Apoptotic cells characterized by loss of cytoplasm, pyknotic nuclei with the appearance of crescent shape or irregularly shaped nuclei along with marginated chromatin were also found. In addition, we observed that some macrophages exhibited cell and organelle swelling which are characteristics of oncosis (Figure 2). Therefore, after interaction with apoptotic neutrophils, the form of macrophage cell death was a mixture of autophagy, apoptosis and oncosis.

\subsection{Confocal microscopy analysis of macrophages after ingestion of apoptotic neutrophils}

To determine the percentages of autophagic, apoptotic and oncotic macrophages, we used 7-AAD (BD Biosciences) to label the apoptotic neutrophils, and MDC/AO, AO and $\mathrm{AO} / \mathrm{EB}$ to stain macrophages after their ingestion of apoptotic neutrophils. As shown in Figure 3, the macrophages contained MDC, AO and EB positive granules with 7-AAD labeled apoptotic neutrophils. Data from three independent experiments showed that MDC positive autophagic macrophages comprised $8.00 \% \pm 2.00 \%$ of the total macrophages. Meanwhile, macrophages with AO positive granules and EB positive cells, indicating apoptotic and oncotic macrophages, comprised $12.33 \% \pm 2.08 \%$ and $3.66 \% \pm 1.50 \%$ of the total macrophages, respectively. Therefore, the predominant forms of phagocytosis-induced macrophage cell death were apoptosis and autophagy.

\section{Discussion}

Clearance of apoptotic cells by the phagocytic process was first demonstrated in mammals and nematodes in the early 1980s [19,20]. However, the steps of phagocytosis of apoptotic cell are still under debate. Cvetanovic and colleagues [21] identified a specific recognition step during macrophage engulfment of apoptotic cells. Hoffmann et al. [22] developed a novel system to distinguish tethering from ingestion during phagocytosis of apoptotic erythrocytes by human monocyte-derived macrophage. DeCathelineau and Henson [23] and Gardai et al. [24] coined the unique term efferocytosis to describe the process of clearance of apoptotic cells. In their efferocytosis model, they proposed three steps of clearance of apoptotic cells; tethering, tickling and internalization. Here, we report for the first time the dynamic process of phagocytosis of human apoptotic neutrophils by RAW264.7 cells. We found that the phagocytosis of human apoptotic neutrophils by RAW264.7 cells could also be divided into recognition and tethering, internalization, digestion and exocytosis stages. These observations
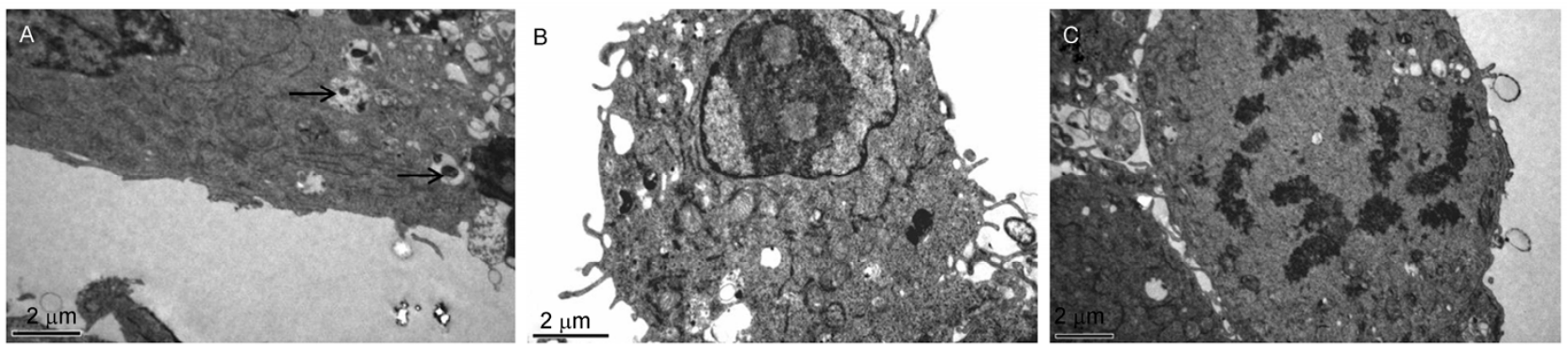

Figure 2 Form of macrophage cell death revealed by transmission electron microscope. Representative micrographs of different forms of macrophage cell death after interaction with apoptotic neutrophils. A, Autophagy. The macrophage was characterized by cell shrinkage, depletion of organelles and autophagosomes $(\rightarrow)$. B, Apoptosis. The macrophage assumed the appearance of irregularly shaped nucleus along with marginated chromatin. C, Oncosis. The macrophage exhibited swelling cellular body and organelles, non-specific fragments of chromatin. 

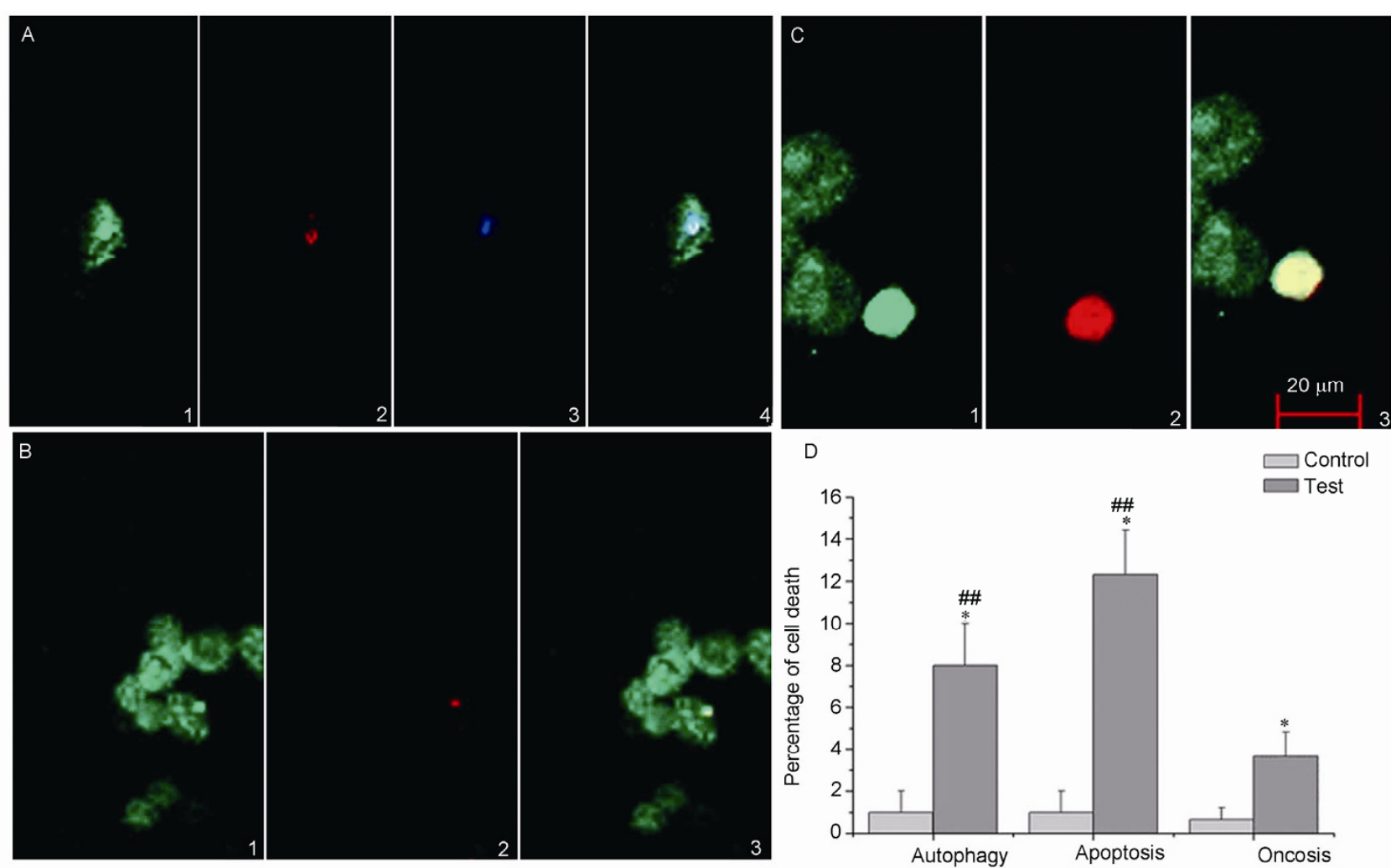

Figure 3 Confocal microscopy analysis of macrophages after ingestion of apoptotic neutrophils. Representative confocal micrographs showing autophagy, apoptosis and oncosis of macrophages after their ingestion of apoptotic neutrophils labeled with 7-AAD. A, The green macrophage stained with AO (1) engulfed an apoptotic neutrophil labeled with 7-AAD (2) produced an autophagosome by MDC staining (3), the merged image appears white in color (4). B, The green macrophage stained with AO (1) which had engulfed a 7-AAD labeled neutrophil (2) showed the presence of green patches of fragmented and condensed chromatin (3). C, Oncotic macrophage stained with AO/EB was visible as a uniform orange color. D, Histogram of the different forms of macrophage cell death. Data are expressed as the mean $\pm \mathrm{SE}(n=3) ;{ }^{*}, P<0.05$ vs. corresponding control group; \#\#, $P<0.05$ vs. oncotic macrophages.

confirmed and extended upon previous studies.

Three types of programmed cell death, autophagy, apoptosis and oncosis, have been well described among other forms of cell death, while necrosis is defined as the sum of changes occurring in the cells after they have died regardless of the prelethal process [25,26]. Phagocytosis-induced apoptosis has been well described in the setting of a bacterial infection [27]. MDC, a well-known specific fluorescent dye that accumulates in acidic vacuoles, is often used as a marker for autophagosomes [28]. In the present study, we used it to determine whether autophagy was an alternative mode of macrophage cell death after ingestion of apoptotic neutrophil. We found that MDC stained the engulfed apoptotic neutrophils and transmission electron microscope confirmed the phenomena. Microtubule-associated protein 1A/1B-light chain 3 (LC3) is a soluble protein and detecting LC3 by immunoblotting or immunofluorescence has become a reliable method for monitoring autophagy and autophagy-related processes, including autophagic cell death. Unfortunately, we failed to measure LC3 to further confirm our findings on phagocytosis-induced autophagy. At the same time, we also found that some macrophages appeared oncotic after they phagocytosed apoptotic neutrophils.
Therefore, we concluded that after ingestion of apoptotic neutrophils, macrophages undergo either apoptosis, autophagy or oncosis. Variation in the form of macrophage cell death may be a result of the different stages of apoptotic cells and the intracellular apoptotic neutrophil load. Alternatively it may be because of the system we used, where the mouse macrophage cell line RAW264.7 ingested human apoptotic neutrophils. Therefore, this area requires further research using primary macrophages to ingest apoptotic cells of the same species. Thus, macrophage cell death after ingestion of apoptotic cells may be a physiological process to enable turnover of terminally differentiated macrophages.

Macrophages play a central role in the control of inflammatory diseases such as asthma, pneumonia and systemic lupus erythematosus (SLE) [29,30]. They are professional scavenger cells for the clearance of apoptotic cells. Our results show that after the ingestion of apoptotic cells, a subset of macrophages undergoes different forms of cell death. Limited macrophage cell death may be beneficial for macrophage turnover and the process of inflammation. Conversely, massive macrophage cell death may be an important factor in the pathogenesis and progression of severe asthma, pneumonia and SLE. Deciphering the mechanisms 
and blocking macrophage cell death after taking up apoptotic neutrophils will provide new insights into the pathogenesis and treatment of these diseases.

Thus, our study measured the kinetics of the phagocytosis of apoptotic neutrophils by macrophages and showed that after ingestion of apoptotic neutrophils, macrophages undergo mostly autophagy and apoptosis. Therefore, macrophage cell death induced by the phagocytosis of apoptotic cells may be a novel mechanism regulating the immune response.

Much gratitude is given to Prof. Zhou Jiang-Ning (School of Life Science, University of Science and Technology of China) for insightful discussions and helpful comments on the manuscript.

1 Kobayashi SD, Voyich JM, Burlak C, DeLeo FR. Neutrophils in the innate immune response. Arch Immunol Ther Exp (Warsz), 2005, 53: 505-517

2 Sladek Z, Rysanek D, Ryznarova H, Faldyna M. Neutrophil apoptosis during experimentally induced staphylococcus aureus mastitis. Vet Res, 2005, 36: 629-643

3 Watson RW, Redmond HP, Wang JH, Bouchier-Hayes D. Bacterial ingestion, tumor necrosis factor-alpha, and heat induce programmed cell death in activated neutrophils. Shock, 1996, 5: 47-51

4 Tauber AI. Metchnikoff and the phagocytosis theory. Nat Rev Mol Cell Biol, 2003, 4: 897-901

5 Djaldetti M, Salman H, Bergman M, Djaldetti R, Bessler H. Phagocytosis - the mighty weapon of the silent warriors. Microsc Res Tech, 2002, 57: 421-431

6 Schagat TL, Wofford JA, Wright JR. Surfactant protein A enhances alveolar macrophage phagocytosis of apoptotic neutrophils. J Immunol, 2001, 166: 2727-2733

7 Fadeel B, Orrenius S, Zhivotovsky B. Apoptosis in human disease: a new skin for the old ceremony? Biochem Biophys Res Commun, 1999, 266: 699-717

8 Choi JJ, Reich CF, Pisetsky DS. The role of macrophages in the in vitro generation of extracellular DNA from apoptotic and necrotic cells. Immunology, 2005, 115: 55-62

9 Odaka C, Mizuochi T, Yang J, Ding A. Murine macrophages produce secretory leukocyte protease inhibitor during clearance of apoptotic cells: implications for resolution of the inflammatory response. J Immunol, 2003, 171: 1507-1514

10 Lichtenberger C, Zakeri S, Baier K, Willheim M, Holub M, Reinisch W. A novel high-purity isolation method for human peripheral blood neutrophils permitting polymerase chain reaction-based mRNA studies. J Immunol Methods, 1999, 227: 75-84

11 Tan BH, Meinken C, Bastian M, Bruns H, Legaspi A, Ochoa MT, Krutzik SR, Bloom BR, Ganz T, Modlin RL, Stenger S. Macrophages acquire neutrophil granules for antimicrobial activity against intracellular pathogens. J Immunol, 2006, 177: 1864-1871

12 Wang C, Wang J, Guo HF, Liu RY. Involvement of Annexin I in the dexamethasone-mediated upregulation of A549 cells phagocytosis of apoptotic eosinophils. Immunol Lett, 2007, 111: 103-110

13 Maderna P, Yona S, Perretti M, Godson C. Modulation of phagocytosis of apoptotic neutrophils by supernatant from dexamethasone-treated macrophages and annexin-derived peptide Ac(2-26). J Immunol, 2005, 174: 3727-3733
14 Hart SP, Alexander KM, Dransfield I. Immune complexes bind preferentially to Fc gamma RIIA (CD32) on apoptotic neutrophils, leading to augmented phagocytosis by macrophages and release of proinflammatory cytokines. J Immunol, 2004, 172: 1882-1887

15 Shi Q, King RW. Chromosome nondisjunction yields tetraploid rather than aneuploid cells in human cell lines. Nature, 2005, 437: 1038-1042

16 Mignot C, Delarasse C, Escaich S, Della Gaspera B, Noé E, Colucci-Guyon E, Babinet C, Pekny M, Vicart P, Boespflug-Tanguy O, Dautigny A, Rodriguez D, Pham-Dinh D. Dynamics of mutated GFAP aggregates revealed by real-time imaging of an astrocyte model of Alexander disease. Exp Cell Res, 2007, 313: 2766-2779

17 Zhu J-h, Horbinski C, Guo F, Watkins S, Uchiyama Y, Chu CT. Regulation of autophagy by extracellular signal-regulated protein kinases during 1-methyl-4-phenylpyridinium-induced cell death. Am J Pathol, 2007, 170: 75-86

18 Guerin PJ, Furtak T, Eng K, Gauthier ER. Oxidative stress is not required for the induction of apoptosis upon glutamine starvation of Sp2/0-Ag14 hybridoma cells. Eur J Cell Biol, 2006, 85: 355-365

19 Savill JS, Wyllie AH, Henson JE, Walport MJ, Henson PM, Haslett C. Macrophage phagocytosis of aging neutrophils in inflammation. Programmed cell death in the neutrophil leads to its recognition by macrophages. J Clin Invest, 1989, 83: 865-875

20 Ellis RE, Jacobson DM, Horvitz HR. Genes required for the engulfment of cell corpses during programmed cell death in Caenorhabditis elegans. Genetics, 1991, 129: 79-94

21 Cvetanovic M, Mitchell JE, Patel V, Avner BS, Su Y, van der Saag PT, Witte PL, Fiore S, Levine JS, Ucker DS. Specific recognition of apoptotic cells reveals a ubiquitous and unconventional innate immunity. J Biol Chem, 2006, 281: 20055-20067

22 Hoffmann PR, deCathelineau AM, Ogden CA, Leverrier Y, Bratton DL, Daleke DL, Ridley AJ, Fadok VA, Henson PM. Phosphatidylserine (PS) induces PS receptor-mediated macropinocytosis and promotes clearance of apoptotic cells. J Cell Biol, 2001, 155: 649-660

23 deCathelineau AM, Henson PM. The final step in programmed cell death: phagocytes carry apoptotic cells to the grave. Essays Biochem, 2003, 39: 105-117

24 Gardai SJ, McPhillips KA, Frasch SC, Janssen WJ, Starefeldt A, Murphy-Ullrich JE, Bratton DL, Oldenborg PA, Michalak M, Henson PM. Cell-surface calreticulin initiates clearance of viable or apoptotic cells through trans-activation of lrp on the phagocyte. Cell, 2005, 123: 321-334

25 Majno G, Joris I. Apoptosis, oncosis, and necrosis. An overview of cell death. Am J Pathol, 1995, 146: 3-15

26 Levin S. Apoptosis, necrosis, or oncosis: what is your diagnosis? A report from the cell death nomenclature committee of the society of toxicologic pathologists. Toxicol Sci, 1998, 41: 155-156

27 Frankenberg T, Kirschnek S, Hacker H, Häcker G. Phagocytosis-induced apoptosis of macrophages is linked to uptake, killing and degradation of bacteria. Eur J Immunol, 2008, 38: 204-215

28 Amer AO, Swanson MS. Autophagy is an immediate macrophage response to legionella pneumophila. Cell Microbiol, 2005, 7: 765-778

29 Zhou H, Kobzik L. Effect of concentrated ambient particles on macrophage phagocytosis and killing of streptococcus pneumoniae. Am J Respir Cell Mol Biol, 2007, 36: 460-465

30 Bhasin M, Wu M, Tsirka SE. Modulation of microglial/macrophage activation by macrophage inhibitory factor (TKP) or tuftsin (TKPR) attenuates the disease course of experimental autoimmune encephalomyelitis. BMC Immunol, 2007, 8: 10

Open Access This article is distributed under the terms of the Creative Commons Attribution License which permits any use, distribution, and reproduction in any medium, provided the original author(s) and source are credited. 